\title{
On the Habitability of Terrestrial Planets in Binary Star Systems
}

\author{
Elke Pilat-Lohinger ${ }^{1}$, Barbara Funk $^{1}$ and Siegfried Eggl ${ }^{2}$ \\ ${ }^{1}$ Institute of Astronomy, University of Vienna, Austria \\ ${ }^{2}$ IMCCE, Paris \\ email: elke.pilat-lohinger@univie.ac.at.
}

\begin{abstract}
The growing number of detected planets in binary star systems requires methods for a quick assessment of possible habitability of planets in such environments. We offer an analytic method to determine habitability of a terrestrial planet in binary star systems. In this context we give an answer to the most important question: Do the radiative and gravitational perturbations of a secondary influence the extent of the habitable zone (HZ)?

After we have defined the borders of the HZ, we will show the dynamical behaviour of a terrestrial planet in the HZ when adding a Jupiter to the system. In such a system the HZ shows signs of mean motion resonances and secular resonances, depending on the architecture of the planetary system.
\end{abstract}

Keywords. Binaries: general, planetary systems, n-body simulations, numerical

\section{Introduction}

The recent discovery of a telluric companion to $\alpha$ Cen B (Dumusque et al., 2012) is a first indication that terrestrial planets can form in multi-stellar systems. Planets that have been detected in binary star systems so far (nearly 60), are however more massive, and can be considered as Jupiters or Neptunes. The fact that more than half of the stars in the Solar neighborhood (Keseleva-Eggleton \& Eggleton, 2001) are in binary or multiple star systems motivates us to study such systems in detail. Planetary motion in binary stars can be either circumstellar (i.e. around one stellar component) or circumbinary (i.e. a far away orbit around both stars). Since most of the detected planets belong to the circumstellar class, we will concentrate on this type in the present study.

Investigations of planets in multi-stellar systems need of course a special treatment, since the stable region, where planetary motion can take place - for the lifetime of the system - is restricted to certain areas due to the gravitational perturbations of the second star. This is more so in tight binary stars (with distances $<100 \mathrm{AU}$ between the two stars) whereas circumstellar motion in wide binaries can be treated similar to planetary motion around a single-star as an approximation - if all celestial bodies are in the same plane.

First general stability studies of planetary motion in binary stars date back to Harrington (1977) which was long before the first planet in a multi-stellar system was detected. While many astronomer were convinced that binary stars could not habour planets, dynamicists showed the contrary (see e.g., Harrington 1977, Dvorak 1984, Rabl \& Dvorak 1988, and others). These studies gained importance due to the discoveries of $\gamma$ Cephei A b, Gliese 86 b, HD41004 A b and caused a renewal of such studies (Holman \& Wiegert 1999, Pilat-Lohinger \& Dvorak 2002, and Pilat-Lohinger et al. 2003).

Of course, not only the evolution of planetary motion in multiple stellar systems is of special interest, so is the formation of planets in such systems, and whether they can 
Table 1. Extension of the stable region of HD41004 A, when $e_{G G}=0.39$ :

\begin{tabular}{l|l|l|l|l|l|}
\hline$e_{b}$ & 0.1 & 0.2 & 0.3 & 0.4 & 0.5 \\
\hline$a_{c}[A U]$ & 4.4 & 4 & 3 & 2.8 & 1.8 \\
\hline
\end{tabular}

habour habitable planets. For both topics, no unique theory exists and further studies are needed to clarify these hotly debated fields of research (see e.g. Müller \& Kley 2012, Batygin et al. 2011, Paadekooper \& Leinhardt 2010, Thébault 2011, Forgan 2012, and references therein).

In this study we analyse the influence of the dynamics on the habitablity of a terrestriallike planet moving in the habitable zone (HZ) of a K2 main sequence star which is part of a tight binary. As a toy model, we used the parameters of the HD41004 system. First we took only the binary star HD41004 AB and determined the HZ of HD41004 A using the method described in Eggl et al. (2012). Then we added the discovered giant planet that orbits the A component at 1.64 AU and perturbs the HZ significantly. Finally, we showed for two orbits of a terrestrial planet in the HZ of HD41004 A possible stable configurations in the 4-body system (binary stars + giant planet + terrestrial planet) for both, low and high eccentricity motions of the binary and the giant planet, respectively.

\section{The HD41004 AB System}

The tight binary star system HD41004 AB is about 43pc away from our Sun. Both stellar components, a $\mathrm{K} 2 \mathrm{~V}$ and an $\mathrm{M} 2 \mathrm{~V}$ star, are accompanied either by a giant planet or by a brown dwarf. Taking the parameters of the binary stars published by Roell et al. (2012), the two masses $0.7 \mathrm{M}_{\text {Sun }}$ (K2V star) and $0.42 \mathrm{M}_{S \text { un }}$ (M2V star) lead to a massratio of 0.38 . While the distance of the two stars seems to be well determined and to be about $20 \mathrm{AU}$, the determination of the binary's eccentricity requires further observations. Table 1 shows the extension of the stable region (defined by $a_{c}$ ) for the K2V star and for different values of the eccentricity of the binary. For the giant planet of the system, we consider an eccentricity of $e_{G G}=0.39$ (Roell et al., 2012).

The given stability borders result from numerical computations using the chaos indicator FLI (introduced by Froeschlé et al., 1997) to distinguish between regular (i.e. periodic and quasiperiodic) and chaotic motion. Following the evolution of the largest tangent vector of a trajectory, chaos is characterized by an exponential increase in the length of this vector, whereas in a regular motion this increase is linear. From table 1, for a binary eccentricity of 0.4 , the stability limit for periodic and quasiperiodic motion is at 2.8 $\mathrm{AU}\left(=a_{c}\right)$. We note that the value of $a_{c}=3.38 \mathrm{AU}$ as reported by Roell $\mathrm{et}$ al. (2012) was calculated using the formula given by Holman \& Wiegert (1999) which has been obtained for a test particle in a circular motion. Our result, however, takes into account the eccentricity of the planet.

Moreover, the high mass of the detected planet HD41004 Ab $\left(M \sin i=2.54 M_{J u p}\right)$ will probably shift the border of stable motion even closer to HD41004 A. A stability study for the tight binary $\gamma$ Cephei (Pilat-Lohinger, 2011) - where the distance of the two stars and their eccentricity is also 20AU and 0.4, respectively - indicates further changes in $a_{c}$ for a 3 Jupiter-mass planet. An extension of these results to HD41004 A will shift the stability border to 2.3 AU. This limit is quite close to the apastron of the detected planet, which for a planetary eccentricity of 0.39 is at approximately $2.28 \mathrm{AU}$. 


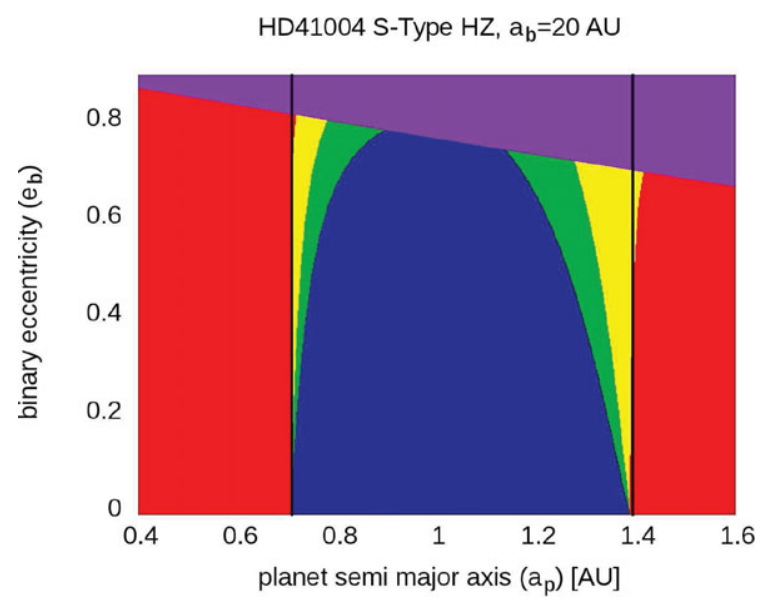

Figure 1. HZ of HD41004 A: The x-axis shows the initial position of the hypothetical terrestial planet and the y-axis denotes different eccentricities of the binary star. The purple area defines the chaotic region of the binary star system. Blue, green and yellow show the different $\mathrm{HZ}$ as defined in the text and red regions indicate areas where the planet is not habitable at all. The two vertical black lines show the borders of the HZ as defined by Kasting et al. (1993).

\section{The Habitable Zone of HD41004 A}

The first investigations on whether binary star systems can habor habitable worlds date back to Huang (1960) and Harrington (1977). In 1993 The numerous discoveries of extra-solar planets since 1995 has made this question one of the hot-topics in astrophysical research. Most investigations on habitability are for single stars, where the opinions differ about the neccessary characteristics to define a planet habitable (see e.g. Kaltenegger et al. 2007, Selsis et al. 2007, Lammer et al. 2009). Recently, Forgan (2012) investigated the effects on the insolation hypothetical planets would receive in such stellar systems. Eggl et al. (2012a) determined the HZ in binary star configurations using analytical expressions as well as numerical simulations, where the results of the two approaches were in good agreement. An application of this study to binary star systems in the solar neigbourhood with distances up to $32 \mathrm{pc}$ showed that 17 out of 19 investigated systems allow dynamically stable terrestrial planets within HZs around at least one of the binary's components (Eggl et al., 2012b).

Using the method desribed in Eggl et al. (2012a) we calculated the HZ of HD41004 A which is shown in fig. 1 .

Since this study takes into account the dynamical effects due to the second star, we distinguish different types of HZ:

Permanent HZ (PHZ; blue region in fig. 1): Because the variations of the planet's eccentricity are small, the entire orbit of the planet is always in the HZ.

Extended HZ (EHZ; green region): The planet is mostly in the $\mathrm{HZ}$ and will leave it only at apo- or peri-astron distances for a short time. In the EHZ, the fluctuations of the planet's eccentricity are higher than in the PHZ.

Averaged HZ (AHZ; yellow region): The orbit of the terrestrial planet in the HZ is highly eccentric due to gravitational perturbations of the secondary so that the planet is outside the HZ for most of the time. However, if the planet's elliptic orbit is replaced by an "averaged" circular orbit with an "averaged" radius $r_{A}$, the planet in the elliptic orbit can be called an "averaged" habitable planet if its averaged circular orbit is in the HZ. Even if the HZ is well inside the stable area of a binary star system, the existence of a 


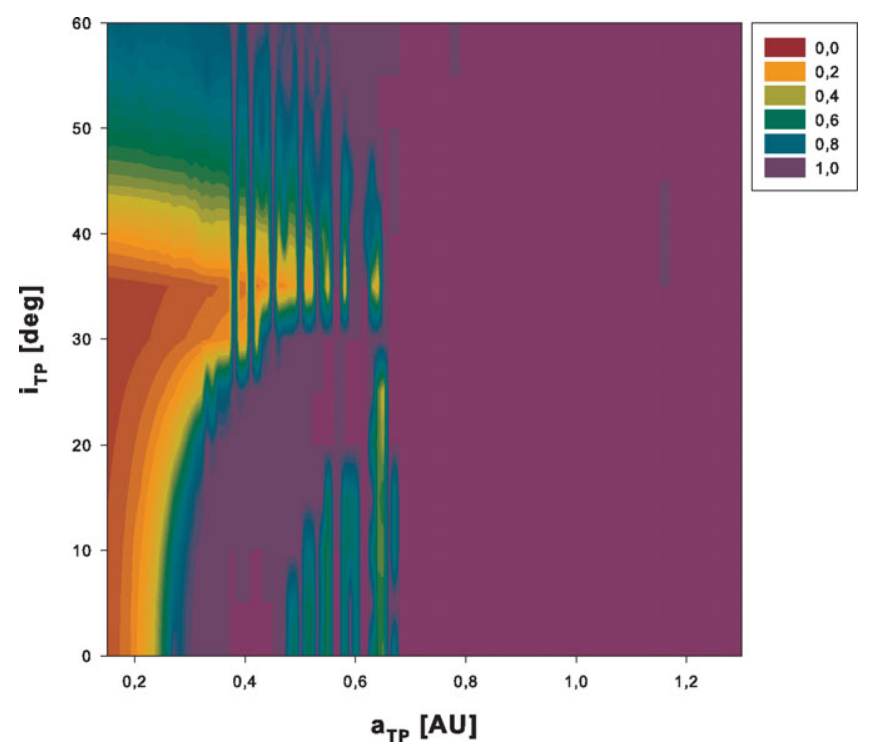

Figure 2. Maximum eccentricity (e-max) map of fictitious terrestrial planets in circumprimary motion in the HD41004 AB system. The $x$-axis shows the initial distance to the primary star and the $y$-axis indicates initial inclinations of the test-planet. Different colors represent different maximum eccentricities of the planetary motion: red corresponds to circular motion and purple depicts escape orbits. Perturbations due to mean motion resonances (vertical stripes) and secular resonances (purple arched band) are clearly visible.

giant planet might change the dynamics so that the planetary motion in the HZ is no longer stable. This can be seen when comparing the region $0.7 \mathrm{AU}<a<1.4 \mathrm{AU}$ of Figs. 1 and 2 .

\section{Perturbations of the $\mathrm{HZ}$}

The gravitational influence of the secondary star and a giant planet on terrestrial-sized test-bodies in circumprimary motion was studied by Pilat-Lohinger in 2005. This author showed that in a multi-planetary system in circumprimary motion in a binary star system, the main perturbations are:

(i) mean motion resonances (MMRs) with the giant planet which result in the appearance of chaotic gaps (see the vertical lines in Fig. 2) in the stable region between the host star and the giant planet; and

(ii) secular perturbations due to interactions between the secondary star and the gas giant which cause a characteristic arch-shape pertrubed band as it can be seen in Fig. 2 . The locations of the resonances depend strongly on the position and the eccentricity of both, the secondary star and the giant planet.

Fig. 2 shows a strong shrinkage in the stable region (red to blue area) for test-planets in circumprimary motion perturbed by a giant planet and the secondary star. In the area between 0.7 and 1.4 AU which from Fig. 1 correspond to the HZ of HD41004 A, the planetary motion is no longer stable even for small values of the eccentricity of the binary system (e.g., $e_{b}=0.2$, in this case). 
Since the observed system of HD41004 A does not allow a stable planet in its HZ, we examined different dynamical configurations of this planetary system which could habor an additional terrestrial planet either at $a_{T P}=0.7 \mathrm{AU}$ (i.e. the inner border of the $\mathrm{HZ}$ given in Fig. 1) or at $1 \mathrm{AU}$ (i.e. right in the middle of the $\mathrm{HZ}$ ).

\section{Dynamical configurations for a stable $\mathrm{HZ}$ of $\mathrm{HD} 41004 \mathrm{~A}$}

The fact that the binary's eccentricity of the HD41004 AB system is not well determined encouraged us to study the habitability of this system for different values of $e_{b}$. To find stable configurations we placed a fictitious terrestrial planet either at $0.7 \mathrm{AU}$ from $\mathrm{HD} 41004 \mathrm{~A}$ or at $1 \mathrm{AU}$, and varied the initial position of the detected gas giant between 1.3 and $5 \mathrm{AU}$ with an eccentricity $e_{G G}=0.39$. In the case of low-eccentricity motion of the binary $\left(e_{b}=0.1\right)$, stable configurations are possible if $a_{G G} \geqslant 3.3 \mathrm{AU}$ for a terrestrial planet at $0.7 \mathrm{AU}$. For $a_{T P}=1 \mathrm{AU}$ no stable configuration was found.

In the case of high eccentricity motion of the binary $\left(e_{b}=0.4\right)$, we did not find any stable configuration for both initial positions of the terrestrial planet. This can be seen from table 1 , which shows the border of stable motion for this binary with $e_{b}=0.4$ at 2.8 $\mathrm{AU}$ and that we had to place the planet at least at $3.3 \mathrm{AU}$ for $e_{b}=0.1$ - which is outside the stable region in the high-eccentric binary system.

\section{Conclusion}

In this study we examined dynamical perturbations of planetary and stellar companions on the habitable zone in a binary star system. Since most detected exo-planets in multi-stellar systems are in circumstellar motion, we restricted our study to this type of planetary motion and took the HD41004 AB system as a model for our computations. Moreover, because we were interested in the HZ of HD41004 A, we simplified this exo-planetary system by ignoring the close-in brown dwarf orbiting HD41004 B. The application of the work by Eggl et al. (2012a) to this binary system (without planet HD41004 A b) indicated that the HZ of HD41004 A is in a distance between 0.7 and 1.4 AU. For circular and low-eccentricity motion of the binary, the entire HZ is not affected by the perturbation of the secondary. A planet in this region will always be in the $\mathrm{HZ}$ (hence calling the HZ a permanent HZ, PHZ). If the eccentricity of the binary is increased, the PHZ will shrink and parts of the HZ will be perturbed where the planet will leave the HZ periodically. Depending on the planetary orbit, these parts of the $\mathrm{HZ}$ are called extended HZ (EHZ) of averaged HZ (AHZ) - for details thereto see Eggl et al. (2012a).

We also included the planet orbiting HDf41004 A at a distance of 1.64 AU in our computations, and showed that the $\mathrm{HZ}$ of the system is completely unstable. That is because the giant planet is too close to the HZ, and moves in a highly eccentric orbit.

Finally, we studied possible stable configurations of this binary for two planets; the detected giant planet and a fictitious terrestrial planet in the HZ. Our FLI computations showed that even if we place the terrestrial planet at $0.7 \mathrm{AU}$ (i.e. the inner border of the HZ) and vary the position of the giant planet, stable configurations were only found for low-eccentricty motion of the binary.

\section{Acknowledgements}

The authors would like to acknowledge the support from the Austrian Science Fund (FWF) - project no. P22603-N16 (EP-L and BF), P20216-N16 (EP-L and SE) and S11608-N16 (EP-L and SE). 


\section{References}

Batygin, K., Morbidelli, A., \& Tsiganis, K. 2011, A\&A, 533, A7

Dumusque, X. et al. 2012, Nature, 492, 207

Eggl, S., Pilat-Lohinger, E., Georgakarakos, N., Gyergyovits, M., \& Funk, B. 2012a, ApJ, 752, 74

Eggl, S., Pilat-Lohinger, E., Funk, B., Georgakarakos, N., \& Haghighipour, N. 2012b MNRAS, 428,3104

Forgan, D. 2012, MNRAS, 422, 1241

Froeschlé, C., Lega, E., \& Gonczi, R. 1997, CMDA, 67, 41

Harrington, R. S. 1977, AJ, 82, 753

Holman, M. J. \& Wiegert P. A. 1999, AJ, 117, 621

Huang, S. S. 1960, PASP, 72, 106

Kaltenegger, L., Traub, W. A., \& Jucks, K. W. 2007, ApJ, 658, 598

\&Kasting, J. F., Whitmire D. P., \& Reynolds, R. T. 1993, Icarus, 101, 108

\&Kiseleva-Eggleton, L., \& Eggleton, P. P. 2001, in Astronomical Society of the Pacific Conferences Series, 229, Evolution of Binary and Multiple Star Systems, Podsiadlowski et al. (eds.), 91

Lammer, H., Bredehöft, J. H., Coustenis, A. et al. 2009, A\& $A R, 17,181$

Müller, T. W. A. \& Kley, W. 2012, A\&̛A, 539, A18

Paardekooper, S. J. \& Leinhardt, Z. M. 2010, MNRAS, 403, L64

Pilat-Lohinger, E. \& Dvorak, R. 2002, CMDA, 82, 143

Pilat-Lohinger, E., Funk, B., \& Dvorak, R. 2003, A\&A, 400, 1085

Pilat-Lohinger, E. 2005, in "Dynamics of Populations of Planetary Systems", Proceedings of IAU Coll. 197, eds. Knezevic Z. and Milani A., Cambridge University Press, 71

Rabl, G. \& Dvorak, R. 1988, A\&A, 191, 385

Roell, T., Neuhäuser, R., Seifahrt, A., \& Mugrauer, M. 2012, A\& $A$, 542, A92

Selsis, F., Kasting, J. F., Levrard, B., et al. 2007, A\&A, 476, 1373

Thébault, P. 2011, CMDA, 111, 29 